\title{
A Pragmatic Study of Basal and Mid-Mixture Insulins as Starter Insulins in Chinese Patients With Type 2 Diabetes: Observations From Long-Term, Real-World Experience
}

\author{
Tianshu Zeng · Huijuan Yuan · Jiangong Ren • Yuanyuan Li • \\ Jianing Hou $\cdot$ Liying Du $\cdot$ Jiankun Zhu $\cdot$ Lulu Chen $\cdot$ Linong Ji
}

Received: November 6, 2020 / Accepted: January 20, 2021 / Published online: February 22, 2021

(c) The Author(s) 2021

\section{ABSTRACT}

Introduction: According to Chinese guidelines, basal insulin (BI) or premixed insulins are recommended insulin starters following the failure of oral antihyperglycemic medication (OAM) in

Supplementary Information The online version contains supplementary material available at https:// doi.org/10.1007/s13300-021-01007-z.

T. Zeng · L. Chen $(\bowtie)$

Department of Endocrinology, Union Hospital, Tongji Medical College, Huazhong University of Science and Technology, Wuhan, China e-mail: cheria_chen@126.com

\section{T. Zeng $\cdot$ L. Chen}

Department of Endocrinology, Hubei Provincial Clinical Research Center for Diabetes and Metabolic Disorders, Wuhan, China

H. Yuan

Department of Endocrinology, Henan Provincial People's Hospital, People's Hospital of Zhengzhou University, Henan, China

J. Ren

Department of Endocrinology, Lanzhou University Second Hospital, Gansu, China

Y. Li $\cdot$ J. Hou $\cdot$ L. Du $\cdot$ J. Zhu

Lilly Suzhou Pharmaceutical Co. Ltd, Shanghai, China

\section{Ji (ه)}

Department of Endocrinology and Metabolism, Peking University People's Hospital, Beijing, China e-mail: jiln@bjmu.edu.cn
Chinese patients with type 2 diabetes (T2D). This pragmatic study investigated the long-term effectiveness, safety, and cost of add-on BI and mid-mixture insulin analog (MMI) regimens in Chinese patients with T2D.

Methods: This multicenter, open-label, pragmatic study randomized patients $1: 1$ to receive either BI or MMI with OAMs adjusted according to current standards of care. We evaluated the change in glycated hemoglobin (HbA1c) from baseline, safety parameters, and antidiabetic medication costs.

Results: Change in HbA1c from baseline showed a statistically greater decrease at week 48 in the MMI group (MMI: $-2.03 \%$ [0.06] vs. BI: $-1.82 \%$ [0.06]; $P<0.05)$. Both groups showed decreases in fasting plasma glucose (mmol/L) (MMI: - 2.53 [0.14] vs. BI: - 3.19 $[0.14] ; \quad P<0.01)$ and postprandial glucose (mmol/L) (MMI: - 4.35 [0.22] vs. BI: -4.33 [0.23]). More patients in the BI group showed increases in OAM use, while OAM use decreased in the MMI group. Both groups showed stable glycemic control with a very limited insulin dose change from week 24 to week 48 . The incidence of total hypoglycemia was higher in the MMI group (MMI: 124\% [30.7] vs. BI: $76 \%$ [18.5], $P<0.0001$ ), but no incidence of severe hypoglycemia was reported in either group. Treatment costs, in terms of average daily cost and cost of glycemic control, were higher in the BI group. 
Conclusion: In long-term real-world use, the MMI and BI groups demonstrated improved glycemic control, with the MMI group showing more significant improvement than the BI group. Hypoglycemia incidence was higher in the MMI group, with no major safety issues through week 48. MMI is likely to provide better price value than $\mathrm{BI}$ for the treatment of T2D in Chinese patients.

Trial Registration: ClinicalTrials.gov identifier: NCT03018938

Keywords: Basal insulin; Chinese patients; Premixed insulin; Real-world study; Type 2 diabetes

\section{Key Summary Points}

Why carry out this study?

According to Chinese guidelines, basal or premixed insulins are recommended insulin starters following oral hyperglycemic medication (OAM) failure in Chinese patients with type 2 diabetes (T2D).

Although several randomized controlled trials (RCTs) have reported on the efficacy and safety of basal, premixed insulins, there is a paucity of data on benefits of long-term treatment with insulin regimens in Chinese patients with T2D.

This pragmatic study investigated the long-term effectiveness, safety, and cost of add-on BI and mid-mixture insulin analog (MMI) regimens in Chinese patients with T2D.

\section{What was learned from the study?}

With 6 months extended treatment, the MMI and BI groups demonstrated improved glycemic control, with the MMI group showing a significantly greater decrease in glycated hemoglobin (HbA1c; MMI: $-2.03 \%$ [0.06] vs. BI: $-1.82 \%$ [0.06]; $P<0.05)$.
There was minimum difference in the glucose control observed between weeks 24 and 48, with limited dose titration and OAM adjustment, indicating treatment inertia.

Overall, in long-term real-world use, the MMI and BI groups demonstrated improved glycemic control, with the MMI group showing more significant improvement combined with fewer OAMs, no major safety issues, and a lower cost, indicating that the treatment is efficacious and relatively safe in Chinese patients with T2D.

\section{DIGITAL FEATURES}

This article is published with digital features, including a summary slide, to facilitate understanding of the article. To view digital features for this article go to https://doi.org/10.6084/ m9.figshare.13580837.

\section{INTRODUCTION}

Type 2 diabetes (T2D) is a chronic metabolic disorder with a wide array of complications that have contributed to mortality and disability worldwide [1]. Globally, the estimated number of people living with diabetes was 463 million (9.3\% of the total world population) in 2019, and is expected to increase to approximately 700 million by 2045 [2]. In China, estimates show that the total number of people with diabetes increased from 20.8 million in 2000 to 109.6 million in 2015 , representing $10.6 \%$ of the total population [3]. This rapid increase could be due to transitions in socioeconomic development, dietary patterns, and behavioral and lifestyle changes that are known to have increased the risk of T2D in China [3].

Comprehensive guidelines for the treatment of T2D have emphasized the importance of glycemic control with appropriate lifestyle 
modifications and oral antihyperglycemic medications (OAMs) [4-6]. According to Chinese guidelines, basal or premixed insulins are recommended insulin starters following OAM failure in Chinese patients with T2D $[4,7,8]$. Premixed insulins contain rapid- and intermediate-acting insulin that control fasting plasma glucose (FPG) and postprandial glucose (PPG) elevations, respectively [9]. In many Asian countries, premixed insulins are more widely used as a starter regimen than basal insulin analog (BI) $[9,10]$.

Several randomized controlled trials (RCTs) have reported on the efficacy and safety of basal and premixed insulins in achieving and maintaining glycemic control in patients with T2D $[11,12]$. Pragmatic trials can provide information about treatment practices because they are conducted in real-life settings and represent unselected patient populations, unlike those seen in RCTs [13]. Insulin treatment in patients with diabetes requires a relatively long treatment period; long-term studies conducted in a real-world setting can provide evidence that informs clinicians and patients about how an intervention performs outside the confines of research settings [13]. These studies provide essential information regarding the safety and effectiveness of a drug in large populations, the drug's economic performance in a naturalistic setting, and the drug's effectiveness in comparison with other treatments [13]. Currently, there is a paucity of data on the benefits of longterm treatment with insulin regimens in Chinese patients with T2D. To understand these benefits in a real-world scenario, in this pragmatic trial, we investigated the long-term treatment effects of basal and premixed insulin in Chinese patients with T2D. Our previously published paper reported 24-week data on the effectiveness and safety of BI or mid-mixture insulin analog (MMI) as insulin starters in Chinese patients with T2D who had uncontrolled blood glucose after OAM treatment [14]. This study reports the long-term (48 weeks) effectiveness and safety as well as cost analyses of BI or MMI with OAM treatment in Chinese patients with T2D in a real-world setting.

\section{METHODS}

\section{Study Design}

This study was a 48-week, open-label, randomized, parallel, two-arm, pragmatic, prospective trial to investigate the effectiveness and safety of $\mathrm{BI}$ and MMI in Chinese patients with T2D uncontrolled by OAMs (Fig. S1 in the Supplemetary Information). The study was conducted across 28 study centers in China. The study design, patient enrollment, and treatment have been described previously in the report on the 24-week results [14]. Here, we report the long-term (48-week) effectiveness and safety results and antidiabetic medication cost analyses. The study was conducted in accordance with the Declaration of Helsinki [15] and the International Conference of Harmonization Good Clinical Practice, and was approved by the participating institutional review boards. A full list of review board committee details is provided in the Supplementary Information. All patients gave their written informed consent before enrollment.

\section{Study Population}

Chinese patients with T2D aged $\geq 18$ years who had been taking at least one OAM and had a glycated hemoglobin (HbA1c) level of $\geq 7.5 \%$ within 12 weeks of study entry were included. The study excluded patients with type 1 diabetes, patients who had received any type of insulin within 24 months of study entry, and patients with a serious preexisting medical condition.

\section{Treatment}

Patients who met the eligibility criteria were randomized 1:1 to receive either BI or MMI with the flexibility to adjust OAMs in line with clinical practice. BI analog is a once-daily longacting insulin (glargine or detemir). MMI ana$\log$ is a $50 / 50$ premixed insulin that contains a $1: 1$ ratio of rapid- and intermediate-acting insulin (lispro 50 or aspart 50; twice-daily dose). OAM use and the insulin dose were initiated, 
titrated, and maintained at the investigators' discretion and based on patient status. The study scheduled 6 visits across the 48-week trial to assess antidiabetic treatment and hypoglycemia, consistent with routine clinical practice. The insulin dose and concomitant OAMs were adjusted by the individual investigator based on the patient's condition and regular clinical practice governed by local guidelines. There were no restrictions on switching or augmenting the initial insulin treatment. Glucose data were collected at baseline, week 24, and week 48 .

\section{Study Endpoints}

Real-world data were analyzed to evaluate glycemic control, including changes in $\mathrm{HbAlc}$, FPG, PPG, and the proportion of patients achieving target HbA1c levels of $<7 \%$. Changes in daily insulin dose, body weight, and OAM usage through 48 weeks were also evaluated, and antidiabetic medication cost analyses (average daily cost and cost of glycemic control) for the 48 weeks were performed. Safety analyses included the incidence rates of total hypoglycemia, nocturnal hypoglycemia, and severe hypoglycemia (an event where assistance is required to administer carbohydrate, glucagon, or other resuscitative actions, and during which the patient may have altered mental status and may require parenteral therapy) from baseline to week 48 .

Cost analyses were based on the Chinese healthcare system perspective at the 2019 price level, and estimated the cost in Chinese yuan (CNY) of antidiabetic medications (MMI, BI, and OAMs) after 48 weeks of treatment. The endpoints of average daily cost included the daily total medication cost associated with OAM and insulin, the daily OAM cost, and the daily insulin cost. Costs associated with glycemic control were also estimated. This included the cost associated with $1 \%$ reduction in HbA1c and the cost per patient of achieving the HbA1c target of $<7 \%$.

\section{Statistical Analyses}

Detailed statistical analyses have been presented previously [14]. At week 48, both effectiveness and safety analyses were performed on randomized patients, and all tests of treatment effects were conducted at a two-sided alpha level of 0.05 . The comparisons of change from baseline to week 48 between treatment arms in HbA1c, FPG, PPG, and body weight were conducted using an analysis of covariance model or a mixed-effects model with repeated measures. Responders for $\mathrm{HbA} 1 \mathrm{c}<7 \%$ at 48 weeks were analyzed by a logistic regression model. Insulin dose was descriptively summarized by treatment group as the total daily dose (IU/kg) with the 2-sample $t$ test $p$ value. OAM usage and incidence rates of hypoglycemic events were compared by Fisher's exact test. Cost analyses were performed on a subset of all randomized patients who had complete information on cost and $\mathrm{HbA} 1 \mathrm{c}$ evaluation. All statistical analyses were performed using SAS System ${ }^{\circledR}$ version 9.4.

\section{RESULTS}

\section{Patient Disposition and Baseline Characteristics}

Overall, 814 patients on OAM treatment were randomized to receive BI $(n=410)$ and MMI $(n=404)$, of whom 663 patients completed the 48-week trial. Patient disposition is shown in Fig. 1. Baseline characteristics were similar for the treatment groups and have been presented previously [14]. Most of the patients in both groups had poorly controlled diabetes with a mean ( \pm standard deviation, SD) HbA1c of $9.8 \pm 1.61 \%$. The mean $( \pm$ SD) duration of diabetes was $9.4 \pm 5.77$ years, the mean $( \pm S D)$ FPG was $11.4 \pm 3.47 \mathrm{mmol} / \mathrm{L}$, and the mean $( \pm$ SD) PPG was $15.8 \pm 4.50 \mathrm{mmol} / \mathrm{L}$. The gender distribution was similar in both groups, with 233 (56.80\%) and 223 (55.20\%) male patients in the BI and MMI groups, respectively. 


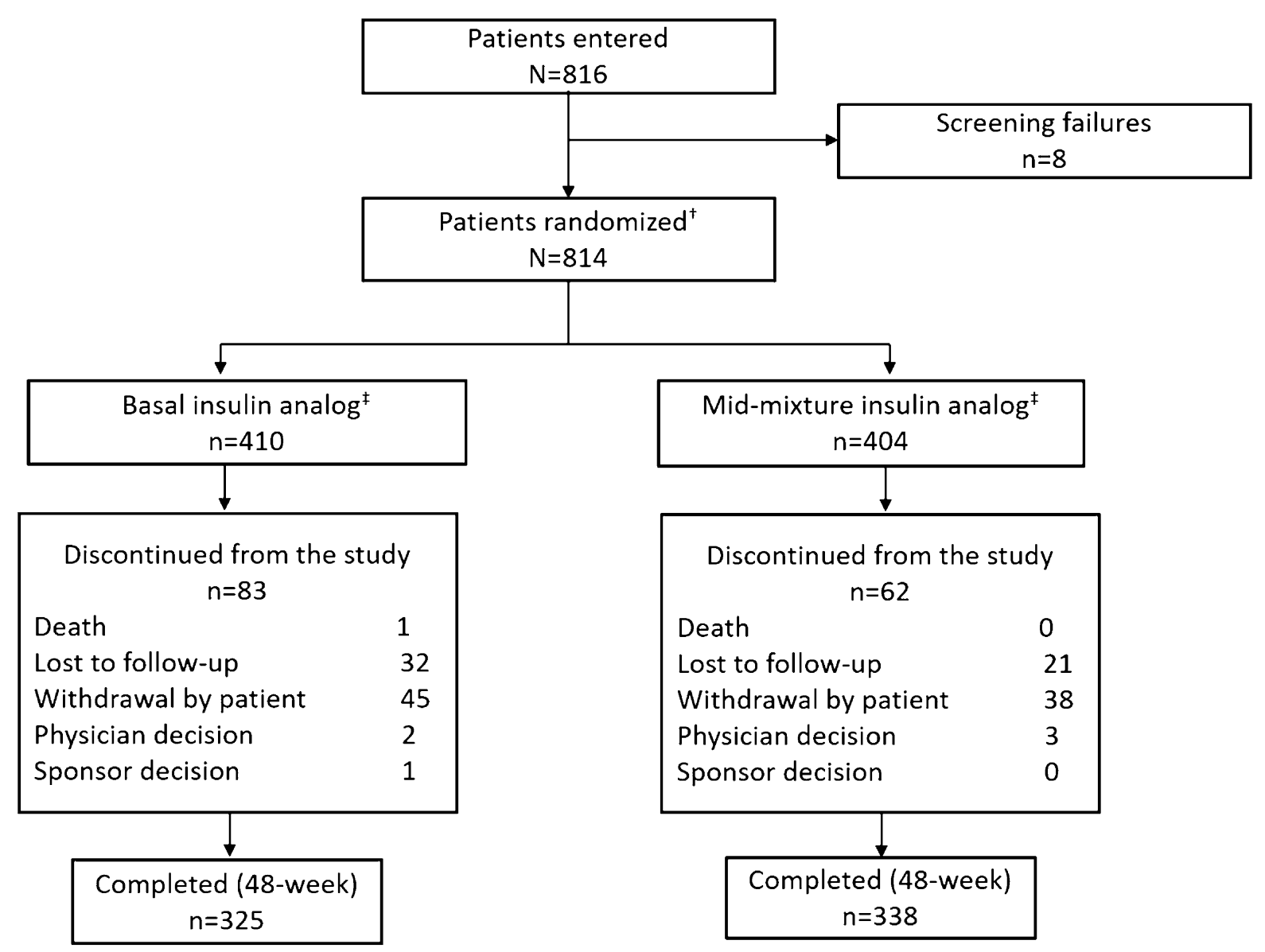

Fig. 1 Patient disposition. ${ }^{\dagger}$ Six patients failed the screening process but were randomized. ${ }^{*}$ Two patients did not have a reason for discontinuation. $N$ total population, $n$ number of patients in each category

\section{Glycemic Control (Baseline to Week 48)}

As reported previously, both the BI and MMI groups demonstrated good glycemic control at week 24 [14]. The least-squares (LS) mean standard error (SE) of the change in HbA1c from baseline to week 24 was similar between the MMI and BI groups (MMI: - 2.15 [0.07] vs. BI -2.00 [0.07]; LS mean difference [95\% CI]: $-0.15 \quad[-0.34,0.04] ; \quad P=0.13)$. With an extended 6 months of treatment, however, a statistically greater decrease in $\mathrm{HbA1c}$ at week 48 was observed in the MMI group (MMI: -2.03 [0.06] vs. BI: - 1.82 [0.06]; LS mean difference $\left[\begin{array}{ll}95 \% & \mathrm{CI}\end{array}\right]$ : $-0.21 \quad[-0.39,-0.03]$; $P<0.05$ ) (Fig. 2) than in the BI group. Similar to the 24-week results, there was a greater reduction in FPG in the BI group than in the MMI group from baseline to week 48 (LS mean [SE]: MMI: -2.53 [0.14] vs. BI: -3.19 [0.14]; $P<0.01$ ), and the reduction in PPG post breakfast was similar in both groups with no significant difference between the groups (LS mean [SE]: MMI: -4.35 [0.22] vs. BI: -4.33 [0.23], $P>0.05)$. The proportion of patients who achieved $\mathrm{HbA1c}<7 \%$ at week 48 was comparable between the groups (MMI: $23.3 \%$ vs. BI: $23.4 \%, P>0.05)$ and was lower compared with week 24 [14].

The changes in these effectiveness parameters after the additional 6 months of treatment showed that there was better glucose control in the MMI group and a limited change in insulin dose from week 24 to week 48 . The mean (SD) dose of BI was 14.6 (7.07) U/day at week 24, with minimal increments to 15.4 (7.09) U/day 


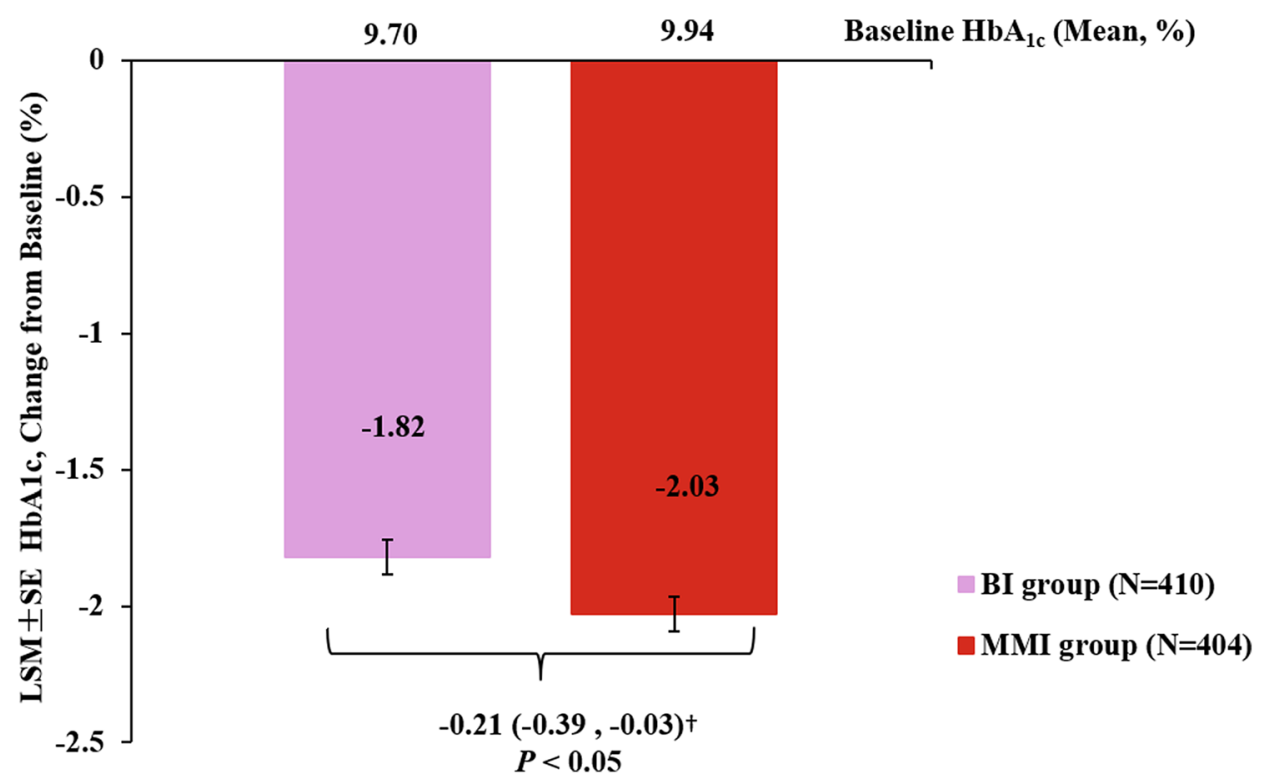

Fig. 2 Changes in HbAlc at week 48 from baseline using analysis of covariance. ${ }^{\dagger}$ LSM difference (95\% CI) between $\mathrm{MMI}+\mathrm{OAM}$ (MMI group) and $\mathrm{BI}+\mathrm{OAM}$ (BI group). LSM values and $P$-values are based on an analysis

at week 48 . The dose of MMI reduced from 24.8 (10.25) U/day at week 24 to 24.2 (11.24) U/day at week 48. There were similar changes in body weight $(\mathrm{kg})$ at week 24 and week 48 in both groups. Table 1 presents the changes in $\mathrm{HbA1c}$, FPG, PPG, and body weight from baseline to week 48 and the daily insulin doses at baseline and week 48 .

A significant intergroup difference in OAM use was detected $(P<0.0001)$ : more patients increased their OAM use in the BI group whereas decreased their OAM use in the MMI group through week 48 (Table 2). The BI group demonstrated increased use of alpha-glucosidase inhibitors, glinides, and insulin secretagogues compared with the MMI group (Table 3). The proportions of patients who discontinued, switched, or intensified insulin treatment through week 48 were comparable between both the groups $(P=0.35$, Table S1 in the Supplementary Information).

\section{Safety Outcomes}

As in the 24-week analysis [14], both BI and MMI showed no major safety issues at week 48. of covariance model. $B I$ basal insulin, $C I$ confidence interval, $H b A I c$ glycated haemoglobin, $L S M$ least-squares mean, $M M I$ mid-mixture insulin, $O A M$ oral antihyperglycemic medication, $S E$ standard error

The overall safety findings were comparable between the groups (Table S2 in the Supplementary Information). No severe hypoglycemia event was reported over the 48 weeks of treatment in either group. The incidence of total hypoglycemia was higher in the MMI group compared with the BI group (MMI: 124\% [30.7] vs. BI: 76\% [18.5], $P<0.0001)$, while the incidence of noctural hypoglycemia was similar between the groups (MMI: 42\% [10.4] vs. BI: 38\% [9.3], $P=0.64$ ) (Table S3 in the Supplementary Information).

\section{Cost Outcomes}

A total of 590 patients (BI: 289, MMI: 301) were included in the cost analysis. During the 48-week treatment period, the mean daily total medication cost per patient per day was $38.5 \%$ higher in the BI group compared with the MMI group (mean [SD], BI: 17.81 [7.07] CNY vs. MMI: 12.86 [5.73] CNY; $P<0.0001)$. Similarly, the mean daily OAM cost per patient per day was $51.0 \%$ higher (mean [SD], BI: 9.71 [6.32] CNY vs. MMI: 6.43 [5.16] CNY; $P<0.0001$ ) and 
Table 1 Changes in glycated hemoglobin, fasting plasma glucose, postprandial glucose, and body weight from baseline to week 48 and the daily insulin dose at baseline and week 48

\begin{tabular}{|c|c|c|c|c|c|}
\hline \multirow[t]{2}{*}{ Parameter } & \multicolumn{2}{|l|}{ BI + OAM } & \multicolumn{2}{|l|}{ MMI + OAM } & \multirow{2}{*}{$\begin{array}{l}\text { Mean difference } \\
\text { Week } 48\end{array}$} \\
\hline & Baseline & Week 48 & Baseline & Week 48 & \\
\hline HbAlc (\%) & $9.70 \pm 1.56$ & $-1.82 \pm 0.06$ & $9.94 \pm 1.65$ & $-2.03 \pm 0.06^{*}$ & -0.21 \\
\hline $\mathrm{FPG}(\mathrm{mmol} / \mathrm{L})$ & $11.31 \pm 3.64$ & $-3.19 \pm 0.14$ & $11.55 \pm 3.30$ & $-2.53 \pm 0.14^{* *}$ & 0.66 \\
\hline PPG (mmol/L) & $15.70 \pm 4.36$ & $-4.33 \pm 0.23$ & $15.87 \pm 4.64$ & $-4.35 \pm 0.22$ & -0.02 \\
\hline Body weight (kg) & $66.87 \pm 11.67$ & $0.64 \pm 0.22$ & $66.89 \pm 10.97$ & $1.46 \pm 0.22^{* *}$ & 0.82 \\
\hline Daily insulin dose (IU/day) & $12.13 \pm 4.37$ & $15.38 \pm 7.09$ & $23.00 \pm 7.68$ & $24.22 \pm 11.24^{* * *}$ & 8.84 \\
\hline
\end{tabular}

Unless otherwise specified, baseline and all daily insulin dose data are presented as the mean \pm SD while other data are presented as the LSM change \pm SE. $P$ values were calculated by comparing the data for the BI and MMI groups at each corresponding time point. ${ }^{*} P<0.05 ;{ }^{* *} P<0.01 ;{ }^{* *} P<0.001$

$B I$ basal insulin, FPG fasting plasma glucose, $H b A l c$ glycated haemoglobin, $L S M$ least-squares mean, $M M I$ mid-mixture insulin, $O A M$ oral antihyperglycemic medication, $P P G$ postprandial glucose, $S D$ standard deviation, $S E$ standard error

the daily insulin cost per patient per day was $25.8 \%$ higher (mean [SD], BI: 8.10 [2.95] CNY vs. MMI: 6.44 [2.46] CNY; $P<0.0001)$ in the BI group.

The total medication cost of a $1 \% \mathrm{HbA} 1 \mathrm{c}$ reduction was $52.9 \%$ higher in the BI group (BI: 3376 CNY vs. MMI: $2208 \mathrm{CNY}$ ). Similarly, for each patient who achieved the HbA1c target of $<7 \%$, the cost was $38.6 \%$ higher in the BI group (BI: 27,010 CNY vs. MMI: 19,484 CNY).

\section{DISCUSSION}

The current study is the first long-term pragmatic study conducted in China to evaluate the effectiveness and safety of BI and MMI as starter insulins in Chinese patients with T2D who had inadequate glycemic control on OAMs. The 24-week results reported that the BI and MMI regimens showed similar improvements in glycemic control in the same population [14]. The results of this 6-months extended treatment showed that MMI provides long-term benefits compared with BI in Chinese patients with T2D, with significantly more decrease in HbA1c. There was a statistically greater decrease in HbA1c levels in the MMI group compared with BI group. The reduction in PPG and proportion of patients who achieved the HbA1c target were comparable between the groups. The decrease in FPG level was consistent with that reported at week 24, with a greater decrease observed in the BI group compared with the MMI group at week 48. These results are consistent with the results from a previous 32-week study that compared the glycemic control achieved with premixed insulin (insulin lispro mixture) twice daily to that with BI (insulin glargine) once daily, both in combination with metformin [16].

Similar to the observations at week 24 , in this 48-week analysis, more patients increased OAM use in the BI group and decreasedOAM use in the MMI group. The OAMs received by patients on the BI regimen combined more glinides, alpha-glucosidase inhibitors, and dipeptidyl peptidase-4 inhibitors to control PPG. We also observed that with additional 6 months of treatment, glucose control and the proportion of patients who achieved the HbA1c target was lower at week 48 compared with week 24. Although there was unsatisfactory glucose control, the insulin dose titrations were very limited, with only 1 to 2 units titrated from week 24 to week 48, indicating treatment inertia in clinical practice.

A large proportion ofpatients with $\mathrm{T} 2 \mathrm{D}$ experience delay in treatment intensification despite having poor glycemic control or failure to achieve the recommended glycemic target. 
Table 2 Oral antihyperglycemic medication usage at baseline and week 48

\begin{tabular}{|c|c|c|c|c|}
\hline & \multicolumn{2}{|l|}{ Baseline } & \multicolumn{2}{|l|}{ Week $48^{a}$} \\
\hline & $\leq 2$ OAMs & $>2 \mathrm{OAMs}$ & $\leq 2$ OAMs & $>2$ OAMs \\
\hline $\mathrm{BI}+\mathrm{OAM}(N=399)$ & $285(71.4)$ & $114(28.6)$ & $274(68.6)$ & $125(31.4)$ \\
\hline \multirow[t]{2}{*}{$\mathrm{MMI}+\mathrm{OAM}(N=398)$} & $291(73.1)$ & $107(26.9)$ & $339(85.2)$ & $59(14.8)$ \\
\hline & Decreased & Same & Increased & Overall $P$ values \\
\hline $\mathrm{BI}+\mathrm{OAM}(N=399)$ & $146(36.6)$ & $157(39.3)$ & $96(24.1)$ & $<0.0001$ \\
\hline $\mathrm{MMI}+\mathrm{OAM}(N=398)$ & $202(50.8)$ & $138(34.7)$ & $58(14.6)$ & NA \\
\hline
\end{tabular}

a Except for the $P$ value, all data are presented as $n(\%) . P$ value: Fisher's exact test

$B I$ basal insulin, $M M I$ mid-mixture insulin, $N$ total number of patients in the specified treatment group with OAM records, $N A$ not applicable, $O A M$ oral antihyperglycemic medication

Table 3 Number of patients using different categories of oral antihyperglycemic medications at week 48

\begin{tabular}{lcc}
\hline OAM category $^{\mathbf{a}}$ & $\begin{array}{l}\text { BI }+ \text { OAM }(\boldsymbol{N}=\mathbf{4 1 0}) \\
\text { Week 48 }\end{array}$ & $\begin{array}{l}\text { MMI + OAM }(\boldsymbol{N}=\mathbf{4 0 4}) \\
\text { Week 48 }\end{array}$ \\
\hline Alpha-glucosidase inhibitors & $191(46.6)$ & $164(40.6)$ \\
Biguanides & $261(63.7)$ & $246(60.9)$ \\
DPP-4 inhibitors & $59(14.4)$ & $45(11.1)$ \\
Glinides & $62(15.1)$ & $22(5.4)$ \\
SGLT-2 inhibitors & $10(2.4)$ & $4(1.0)$ \\
Sulfonylureas & $130(31.7)$ & $70(17.3)$ \\
Thiazolidinediones & $19(4.6)$ & $28(6.9)$ \\
\hline
\end{tabular}

${ }^{a}$ Data are presented as $n(\%)$

$B I$ basal insulin, $D P P$ dipeptidyl peptidase, $N$ total number of patients in the specified treatment group, $M M I$ mid-mixture insulin, $n$ number of patients in the specified category, OAM oral antihyperglycemic medication, SGLT sodium-glucose cotransporter

This failure to intensify treatment in a timely manner is termed clinical or treatment inertia [17-19]. In China, the real-world ORBIT study reported a similar situation with lack of insulin adjustment in which only a $0.03 \mathrm{IU} / \mathrm{kg}$ increase in the mean daily dose of BI was reported over 6 months of treatment [20]. Particularly in China, treatment inertia in routine clinical practice can be due to various reasons, including the economic burden of insulin therapy and the costs associated with SMBG monitoring, nonproficiency in self-management of insulin therapy, and a lack of communication between the patient and physician [20].
From a safety perspective, we observed that total hypoglycemia was higher in the MMI group compared with the BI group, consistent with the 24-week analysis [14] and across other studies $[21,22]$, including findings from a previous systematic research that reported an increase in overall hypoglycemia with premixed insulin analogs [23] and a literature review that reported a higher incidence of hypoglycemic events in real-world studies compared to RCTs [24]. Of note, during the 48-week treatment period, no severe hypoglycemiawas observed and the incidence of nocturnal hypoglycemia was similar between both groups, indicating 
long-term safety with insulin therapy. Considering that to date there is limited evidence supporting the impact of insulin therapy on hypoglycemia, the overall safety findings from this long-term real-world study based on clinical practice in China report meaningful observations and are of clinical importance.

Along with effectiveness and safety, cost has become an important factor in healthcare decision-making. The average daily total costs associated with OAM and insulin were significantly higher with BI compared with MMI. In addition, the overall medication cost to achieve the HbA1c target was found to be higher in the BI group. This could be because the unit price for $\mathrm{BI}$ is higher than that for MMI and patients on BI combined more OAMs to achieve glycemic control. Overall, the results suggest that an MMI regimen is cost-saving and likely to provide better value for money than the BI regimen for the treatment of T2D in China.

\section{Limitations}

As this was a real-world study with very limited visits and control, fasting glucose and 2-h PPG levels were only tested after breakfast, so glucose control and glucose fluctuations throughout the day could not be reported. Secondly, analysis between the groups were based on the original group; that is, the treatment at randomization. In a real-world setting, insulin treatment could be changed, with some patients initiated on BI may have changed to premixed insulin treatment and vice versa. Also, the cost analysis reported in this study was a post-hoc analysis that included the direct medical cost but not the indirect cost of hypoglycemia. Therefore, the results need to be interpreted with caution, and further analyses with similar regimens in patients with T2D may be worth conducting.

\section{CONCLUSIONS}

This real-world study reported that both BI and MMI significantly improved glycemic control in Chinese patients with T2D over 48 weeks with no major safety issues for a long treatment period. However, there was minimum difference in glucose control between weeks 24 and 48 , with limited dose titration and OAM adjustment. When compared with BI, MMI showed a greater improvement in glycemic control combined with fewer OAMs and a lower cost, indicating that the treatment is efficacious and relatively safe in Chinese patients with T2D.

\section{ACKNOWLEDGEMENTS}

We thank the participants of the study. The authors would like to thank Li Zhou from Lilly Suzhou Pharmaceutical Co. Ltd. for data analysis and interpretation support (funded by Eli Lilly and Company).

Funding. This study was funded and supported by Eli Lilly and Company. Medical writing assistance support and the journal's rapid service fee for this publication was funded by Eli Lilly and Company.

Medical Writing and/or Editorial Assistance. The authors would like to thank Deepika Kajarekar from Syneos Health for medical writing support. Support for this assistance was funded by Eli Lilly and Company.

Authorship. All named authors meet the International Committee of Medical Journal Editors (ICMJE) criteria for authorship for this article, take responsibility for the integrity of the work as a whole, and have given their approval for this version to be published.

Author Contributions. YL: conception of work and data interpretation; HY and JR: data collection and interpretation; LJ and LC: acquisition and interpretation of data; LD and JZ: analysis and data interpretation. All authors were involved in the critical revision of the paper.

Prior Presentation. The contents from this manuscript were previously presented at an American Diabetes Association meeting; see Chen L et al. Diabetes 2020 Jun;69(Suppl. 1), 
available at https://doi.org/10.2337/db20-2190PUB.

Disclosures. The author Tianshu Zeng is employed as a professor in a government unit in China. The authors Huijuan Yuan, Jiangong Ren, Linong Ji, and Lulu Chen declare that they have no affiliations with or involvement in any organization or entity with any financial interest or nonfinancial interest in the subject matter or materials discussed in this manuscript. The authors Yuanyuan Li, Jianing Hou, Liying Du, and Jiankun Zhu are full-time employees of Lilly Suzhou Pharmaceutical Co. Ltd., Shanghai, China.

Compliance with Ethics Guidelines. Institutional ethics committee approval was obtained for the study, and written informed consent was taken from each patient before participation. A full list of review board committee details is provided in the Supplementary Information. The study was conducted in accordance with the Declaration of Helsinki and the International Conference of Harmonization Good Clinical Practice, and was approved by the participating institutional review boards.

Data Availability. The datasets generated and/or analyzed during the current study are available from the corresponding author on reasonable request.

Open Access. This article is licensed under a Creative Commons Attribution-NonCommercial 4.0 International License, which permits any non-commercial use, sharing, adaptation, distribution and reproduction in any medium or format, as long as you give appropriate credit to the original author(s) and the source, provide a link to the Creative Commons licence, and indicate if changes were made. The images or other third party material in this article are included in the article's Creative Commons licence, unless indicated otherwise in a credit line to the material. If material is not included in the article's Creative Commons licence and your intended use is not permitted by statutory regulation or exceeds the permitted use, you will need to obtain permission directly from the copyright holder. To view a copy of this licence, visit http://creativecommons.org/licenses/by$\mathrm{nc} / 4.0 /$.

\section{REFERENCES}

1. Zheng Y, Ley SH, Hu FB. Global aetiology and epidemiology of type 2 diabetes mellitus and its complications. Nat Rev Endocrinol. 2018;14:88-98.

2. International Diabetes Federation. IDF diabetes atlas, 9th edition. 2019. https://www.diabetesatlas. org/upload/resources/material/20200302_133351_ IDFATLAS9e-final-web.pdf. Accessed 6 Mar 2020.

3. Foos V, Wang $\mathrm{K}$, McEwan $\mathrm{P}$, et al. Assessing the burden of type 2 diabetes in China considering the current status-quo management and implications of improved management using a modeling approach. Value Health Reg Issues. 2019;18:36-46.

4. Jia W, Weng J, Zhu D, et al. Standards of medical care for type 2 diabetes in China 2019. Diabetes Metab Res Rev. 2019;35:e3158.

5. American Diabetes Association. Pharmacologic approaches to glycemic treatment: standards of medical care in diabetes-2019. Diabetes Care. 2019;42(Suppl 1):S90-102.

6. American Diabetes Association. Cardiovascular disease and risk management: standards of medical care in diabetes-2019. Diabetes Care. 2019;42(Suppl 1):S103-23.

7. Wang C, Gao Y, Zhu L, Huang M, Wu Y, Xuan J. Treatment patterns in patients with newly diagnosed type 2 diabetes in China: a retrospective, longitudinal database study. Clin Ther. 2019;41: 1440-52.

8. Tien KJ, Hung YJ, Chen JF, et al. Basal insulin therapy: unmet medical needs in Asia and the new insulin glargine in diabetes treatment. J Diabetes Investig. 2019;10:560-70.

9. Su Q, Liu C, Zheng H, et al. Comparison of insulin lispro mix 25 with insulin lispro mix 50 as insulin starter in Chinese patients with type 2 diabetes mellitus (CLASSIFY study): subgroup analysis of a phase 4 open-label randomized trial. J Diabetes. 2017;9:575-85.

10. Kumar A. Efficacy and safety of biphasic insulin aspart and biphasic insulin lispro mix in patients with type 2 diabetes: a review of the literature. Indian J Endocrinol Metab. 2016;20:288-99. 
11. Sheu WH, Ji L, Lee WJ, Jabbar A, Han JH, Lew T. Efficacy and safety of premixed insulin analogs in Asian patients with type 2 diabetes: a systematic review. J Diabetes Investig. 2017;8:518-34.

12. Anyanwagu U, Mamza J, Gordon J, Donnelly R, Idris I. Premixed vs basal-bolus insulin regimen in type 2 diabetes: comparison of clinical outcomes from randomized controlled trials and real-world data. Diabet Med. 2017;34:1728-36.

13. Blonde $\mathrm{L}$, Marre $\mathrm{M}$, Vincent $\mathrm{M}$, et al. Insulin regimens and glycemic control in different parts of Europe over 4 years after starting insulin in people with type 2 diabetes: data from the CREDIT noninterventional study. Diabetes Res Clin Pract. 2017;133:150-8.

14. Zhang X, Ma Y, Chen H, Lou Y, Ji L, Chen L. A pragmatic study of mid-mixture insulin and basal insulin treatment in patients with type 2 diabetes uncontrolled with oral antihyperglycaemic medications: a lesson from real-world experience. Diabetes Obes Metab. 2020;22:1436-42.

15. World Medical Association. Declaration of Helsinki: Recommendations guiding physicians in biomedical research involving human subjects. JAMA. 1997;277:925-6.

16. Malone JK, Bai S, Campaigne BN, Reviriego J, Augendre-Ferrante B. Twice-daily pre-mixed insulin rather than basal insulin therapy alone results in better overall glycaemic control in patients with type 2 diabetes. Diabet Med. 2005;22:374-81.

17. Khunti K, Wolden ML, Thorsted BL, Andersen M, Davies MJ. Clinical inertia in people with type 2 diabetes: a retrospective cohort study of more than 80,000 people. Diabetes Care. 2013;36:3411-7.

18. Brown JB, Nichols GA, Perry A. The burden of treatment failure in type 2 diabetes. Diabetes Care. 2004;27:1535-40.
19. Khunti K, Nikolajsen A, Thorsted BL, Andersen M, Davies MJ, Paul SK. Clinical inertia with regard to intensifying therapy in people with type 2 diabetes treated with basal insulin. Diabetes Obes Metab. 2016;18:401-9.

20. Ji L, Zhang P, Zhu D, et al. Observational Registry of Basal Insulin Treatment (ORBIT) in patients with type 2 diabetes uncontrolled with oral antihyperglycaemic drugs: real-life use of basal insulin in China. Diabetes Obes Metab. 2017;19:822-30.

21. Malone JK, Kerr LF, Campaigne BN, Sachson RA, Holcombe JH, Lispro Mixture-Glargine Study Group. Combined therapy with insulin lispro Mix $75 / 25$ plus metformin or insulin glargine plus metformin: a 16-week, randomized, open-label, crossover study in patients with type 2 diabetes beginning insulin therapy. Clin Ther. 2004;26: 2034-44.

22. Buse JB, Wolffenbuttel BH, Herman WH, et al. DURAbility of basal versus lispro mix 75/25 insulin efficacy (DURABLE) trial 24-week results: safety and efficacy of insulin lispro mix 75/25 versus insulin glargine added to oral antihyperglycemic drugs in patients with type 2 diabetes. Diabetes Care. 2009;32:1007-13.

23. Ilag LL, Kerr L, Malone JK, Tan MH. Prandial premixed insulin analog regimens versus basal insulin analog regimens in the management of type 2 diabetes: an evidence-based comparison (review). Clin Ther. 2007;29:1254-70.

24. Elliott L, Fidler C, Ditchfield A, Stissing T. Hypoglycemia event rates: a comparison between realworld data and randomized controlled trial populations in insulin-treated diabetes. Diabetes Ther. 2016;7:45-60. 